\title{
Sulla variazione del punto di fusione con la pressione
}

\author{
P. E VALIJE
}

\section{INTRODIZIONE.}

Il processo della fusione è un ambiamento di stato, regolato dall'equazione termodinamica di Clapeyron

$$
\frac{d T_{s}}{d p_{s}}=\frac{\Delta V}{\int S}
$$

Una semplice interpretazione cinetica del processo della fusione è stata data, parecchio tempo fa, da F. A. Lindmamn (1).

Secondo Lindmann la fusione di un eristallo avviene quando l'ampiezza delle oscillazioni termiche degli atomi diventa abbastanza grande da far entrare in collisione le "sfere d'azione" degli atomi stessi.

In una precedente nota $\left(^{3}\right)$ è stato osservato che tali collisioni si devono riferire alle oscillazioni termiche trasversali, poichè, nel passaggio dalla fase solida alla fase liquida, si verifica una brusca diminuzione della rigiditì del cristallo.

Tenuto conto di queste osservazioni e dell'espressione della frequenza massima delle oscillazioni termiche trasversali $\left(^{2}\right)$, la relazione di Lindmamn puo essere scritta nella forma

$$
\frac{T_{s}}{T_{o s}}=\left(\frac{\varrho_{o s}}{\varrho_{s}}\right)^{2 / 3} e^{2 \gamma_{o t s}\left(1-\frac{\varrho_{o s}}{\varrho_{s}}\right)}
$$

in cui $\gamma_{o t s} \dot{e}$ il parametro relativo alle predette oscillazioni, valutato alla densità $o_{o s}$.

La [2] lega la temperatura di fusione $T_{s}$, alla densità della fase solida os al punto di fusione.

Come è stato gia posto in evidenza $\left({ }^{3}\right)$, la temperatura di fusione presenta un massimo per $\varrho_{s}=3 \gamma_{o t s} \varrho_{s s}$. Quindi la fusione può avvenire a qualsiansi temperatura, purchè la pressione sia tale da rendere sufficientemente piccola la distanza media tra. gli atomi.

Il calcolo della temperatura di fusione $T_{s}$, in funzione della pressione $p_{s}$, richiede peraltro la conoscenza dell'equazione di stato. Tella nota sopracitata $\left({ }^{3}\right)$, mediante la formula di Lindmann nella forma [2] e un'equazione di stato per i solidi precedentemente stabilita $\left({ }^{2}\right)$, l'Autore ha ottenuto, tra l'altro, la relazione

$$
d T_{s}=\frac{2 \frac{T_{s}}{K_{T}}\left(\gamma_{o t s} \frac{\varrho_{o s}}{\varrho_{s}}-1 / 3\right)}{1+2 \alpha_{o} K_{o T} \frac{T_{s}}{T_{T}}\left(\gamma_{o t s} \frac{\rho_{o s}}{\varrho_{s}}-1 / 3\right)}
$$

nella quale $K_{T}$ è l'incompressibilità isoterma al punto di fusione, ed $\alpha_{0}$ la dilatazione temica in condizioni ordinarie.

Il confronto della [3] con i risultati spepimentali, a pressione nomale, è stato eseguito soltanto per il ferro $\left(^{3}\right)$. In questa nota il confronto viene esteso al litio, sodio e potassio.

\section{RICIIIAI AD ALCUNE RELAZIONI DELLA TEORIA DEI SOLIDI.}

ì opportuno ricordare alcune relazioni contenute nelle note già (itate $\left({ }^{2}\right),\left({ }^{3}\right)$.

Se si indiano con $V_{o l}$ e $V_{o l}$, le velocità delle onde longitudinali e trasversali alla densità $g_{\theta}$, con $\gamma_{o l}$ e $\gamma_{o^{\prime}}$ i relativi parametri alla stessa densità, l'incompressibilità isoterma $K_{T}$, per temperature superiori alle temperature carateristiche, è data da

$$
K_{T}=K_{o T}\left(\frac{\varrho}{\varrho_{o}}\right)^{1 / \omega} e^{2 \Gamma\left(1-\frac{\varrho_{0}}{\varrho}\right)}
$$


nellat quale

$$
\Gamma=\gamma_{o l}+\frac{a^{2}}{1-a^{2}}\left(\gamma_{o l}-\gamma_{o t}\right)
$$

coll

$$
a^{2}=\frac{4}{3} \frac{V_{o l}^{2}}{V_{o l}^{2}} \simeq \frac{4}{9}
$$

Detto inoltre $C_{v}$ il calore specifico a volume costante e a la dilatazione termica si ha

nella quale

$$
\alpha K_{T}=\gamma \varrho O_{v}
$$

$$
\gamma=\frac{\gamma_{l}+2 \gamma_{\ell}}{3}
$$

e risulta

$$
\begin{aligned}
& \gamma_{\iota} \varrho=\text { cost. } \\
& \gamma_{\iota} \varrho=\text { cost. }
\end{aligned}
$$

In condizioni ordinarie, purchè la temperatura non sia inferiore alle temperature caratteristiche, la [6] fornisce

$$
\gamma_{o}=\frac{c_{s} K_{o T} A}{3 R o_{n}}
$$

dove $A$ è il peso atomico.

Combinando poi la [5] e la [7] si ottiene

$$
\begin{gathered}
\gamma_{o l}=\frac{3 \gamma_{o} b+2 \Gamma}{2+3 b} \\
\gamma_{o t}=\frac{3 \gamma_{o}(1+b)-\Gamma}{2+3 b}
\end{gathered}
$$

in cui

$$
b=\frac{a^{2}}{1+a^{2}} \simeq \frac{4}{5}
$$

Dalla seconda dlelle [8] si ricava infine

$$
\gamma_{o t s}=\frac{Q_{0}}{\rho_{n \mathrm{~s}}} \gamma_{o t}
$$

Espressione approsstalita di $d T_{s} / d p_{s} \quad A$ PRESSIONE ORDINARIA.

Il confronto della [3] con i risultati sperimentali viene effettuato a pressione ordinarial.

D'or"a in avanti verrà pertanto indicata con $Q_{0}$ la densità della fase solida a temperatura $T_{0}$ e a pressione ordinaria, con $Q_{o s}$ la densità della fase solida alla temperatulat di fusione $T_{o s}$ a pressione ordinaria, con $K_{o T}$ l'incompressibilità isoterma alla densità $\varrho_{0}$, con $K_{T}$ l'incompressibilità isoterma alla densita $Q_{o s}$, con $a_{0}$ la dilatazione termica alla densità $Q_{0}$, e con $\gamma_{o t s}$ il parametro delle onde trasversali alla densità $\varrho_{o s}$.

La temperatura $T_{o}$ è l'ordinaria temperatura rli laboratorio.

Posto nella [3] $\varrho_{s}=\varrho_{o s}$, si ha

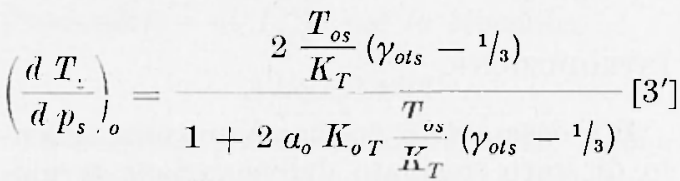

Invere di calcolare gos mediante l'equazione di stato, come è stato fatto per il ferro nella nota precedente $\left({ }^{3}\right)$, si può scrivere, con sufficiente approssimazione,

$$
\varrho_{o s} \simeq \frac{\varrho_{o}}{1+\alpha_{o}\left(T_{o s}-T_{o}\right)}
$$

Conviene inoltre sviluppare $K_{T}$. Ialla [4] si ottiene

$K_{T}=K_{o T}+\frac{K_{o T}}{\varrho_{o}}(1 / 3+2 \Gamma)\left(\varrho_{o s}-\varrho_{o}\right)+\ldots$

Introducendo la [12] e la [13] nella [3'], con qualche passaggio si ha

$\left(\frac{d T_{s}}{d p_{s}}\right)_{o}=2\left(\gamma_{o t s}-1 / 3\right) T_{o s} \frac{1}{K_{o T}} \frac{1+e-d}{1+e}$

in rui

$$
\begin{gathered}
d=\frac{a_{o}(2 \Gamma+1 / 3)\left(T_{o s}-T_{o}\right)}{1+a_{o}\left(T_{o s}-T_{o}\right)} \\
e=2 a_{o} T_{o s}\left(\gamma_{o t s}-1 / 3\right)
\end{gathered}
$$

E questa man relazione approssimata che consente di calcolare facilmente $\left(d T_{s} / d p_{s}\right)_{0}$.

\section{COYFRONTO COY I RISULTATI SPERLAENTALI}

Nella tabella I sono contenuti i dati sperimentali relativi agli elementi litio, sorlio e potassio.

Le temperature di fusione, in funzione 
della pressione, e le relative variazioni di volume tra la fase liquida e la fase solida, costituiscono i risultati delle misure eseguite da P. W. Bridgman ('), mentre le entropie di fusione sono state declotte dai calori latenti di fusione contenuti nell'Handbook of Chemistry and Physies (5).
Jar tabella II contiene i valori delle grandezze che intervengono nella [3"']. Le grandezze $2 \Gamma$ e $K_{o T}$ sono state precedentemente valutate dallo serivente $\left({ }^{2}\right)$, $\left({ }^{3}\right)$. Il calcolo dei valori di $\gamma_{0 t s}$ is stato invece effettuato mediante le relazioni [9], [10], [11] e [12].

'Tabella I

\begin{tabular}{|c|c|c|c|c|}
\hline \multirow{2}{*}{ Elemento } & \multicolumn{4}{|c|}{ Pressione } \\
\hline & 1 & 4000 & 8000 & 12.000 \\
\hline \multicolumn{5}{|l|}{$7 i$} \\
\hline$t_{o s}$ & $178^{\circ}, 4$ & $191^{\circ}, 8$ & 2010,6 & - \\
\hline \multicolumn{5}{|l|}{$N a$} \\
\hline tos & $97^{\circ}, 62$ & $128^{\circ}, 8$ & $155^{\circ}, 1$ & $177^{\circ}, 5$ \\
\hline $1 V^{\prime}$ & 0,02787 & 0,02072 & 0.01711 & 0,01398 \\
\hline$\Delta S$ & 0,358 & - & - & - \\
\hline \multicolumn{5}{|l|}{ K } \\
\hline tos & 620,5 & $115^{\circ}, 8$ & $152^{\circ}, 5$ & $179^{\circ}, 6$ \\
\hline$A \mathrm{~V}^{\mathrm{r}}$ & 0,02680 & 0,01676 & 0,01073 & 0,00642 \\
\hline$\Delta N^{2}$ & 0,196 & - & - & - \\
\hline $\begin{array}{l}\text { Le pr } \\
\text { variazioni }\end{array}$ & $\begin{array}{l}\text { sono il } \\
\text { me } A 1 \text { in }\end{array}$ & $\begin{array}{l}11^{-2}, \text { le } t \\
\text { le entro }\end{array}$ & $\begin{array}{l}\text { ature di } \\
\text { usione } d x\end{array}$ & $\begin{array}{l}t_{o s} \text { in }{ }^{\circ} \mathrm{C}, \text { le } \\
\text { le gratili }{ }^{-1} g^{-1} \text {. }\end{array}$ \\
\hline
\end{tabular}

Tabella II

\begin{tabular}{|c|c|c|c|c|c|c|}
\hline Elemento & $\Lambda$ & $\varrho_{0}$ & $\alpha_{0}$ & $K_{o} T$ & $2 \Gamma$ & Yots \\
\hline$l i$ & 6,940 & 0,534 & $1,810^{-4}$ & 11,67101 & 3,645 & 0,931 \\
\hline$N a$ & 22,997 & 0,971 & $2,1610^{-1}$ & $6,2610^{1}$ & 4,345 & 1,072 \\
\hline$\kappa$ & 39,096 & 0.87 & $2,5 \mathrm{I} 0^{-4}$ & $2,9110^{1}$ & 4,526 & 1,075 \\
\hline$F e$ & 55,85 & 7,87 & $3,3610^{-5}$ & $1,71+10^{6}$ & 3,784 & 1,625 \\
\hline
\end{tabular}

Le densita $\varrho_{0}$ sono in $\mathrm{cm}^{-3}$, le dilatazioni termiche $\alpha_{0}$ in gradi-1, le incompressibilita isoterme $\kappa_{o T}$ in $\mathrm{K} \ddot{m} \mathrm{~cm}^{-2}$. 
Per il ferro si è posto $T_{\text {os }}=1808^{\circ} \mathrm{K}$ e si ì assunto $T_{0}=293^{\circ} \mathrm{K}$.

I rati contenuti nelle tabelle I e II consentono di eseguire un confronto fra i dati sperimentali e la teoria.
I calori teorici ottenuti per il litio, sortio, potassio e ferro, vengono confrontati con $i$ dati sperimentali.

$L$ 'accordo risulta abbastanza soddisfacente.

Tabella II I

\begin{tabular}{|c|c|c|c|c|}
\hline \multirow{3}{*}{ Elemento } & \multicolumn{4}{|c|}{$\left(d T_{s} d_{s} p\right)^{\circ}$ in gradi/lo $0^{3} \mathrm{Kg}\left(\mathrm{m}^{-2}\right.$} \\
\hline & \multicolumn{2}{|c|}{ Valori sperimentali } & \multicolumn{2}{|c|}{ Valori teorici } \\
\hline & $\begin{array}{l}\text { Dalla relazione } \\
\text { di ('lapeyron }\end{array}$ & $\begin{array}{l}\text { Dai dati di } \\
\text { P.W. Bridgman }\end{array}$ & $\begin{array}{c}\text { Calcolo } \\
\text { rigoroso }\left({ }^{3}\right)\end{array}$ & $\begin{array}{c}\text { Calcolo } \\
\text { approssimato } \\
\text { mediante la! } 3 "\end{array}$ \\
\hline$L_{i} i$ & - & 3,8 & - & 4,7 \\
\hline$N a$ & 7,7 & 8,5 & - & 8,4 \\
\hline$\kappa$ & 13,4 & 16,0 & - & 15,9 \\
\hline$F e$ & 2,9 & - & 2,9 & 2,8 \\
\hline
\end{tabular}

Nella prima colonna della tabella III sono elencuti i valori di $\left(d T s / d p_{s}\right)_{0}$ ottenuti per interpolazione dai dati di P. W. Bridgman; la seconda colonna contiene gli analoghi valori ottenuti dalla formula di Clapeyron. Giova avvertire che, mentere per il sorlio e il potassio sono state utilizzate le entropie di fusione e le variazioni di volume contenute nella tabella $I$, il dato per il ferro is stato valutato da F. Simon $\left(^{8}\right)$.

Nella terza colonna della tabella III è riportato il valore teorico di $\left(d T_{s} / d l_{s}\right)_{o}$ per il ferro ottenuto, nella nota precerlente $\left({ }^{3}\right)$, mediante il adcolo rigoroso della [3].

La quarta colonna infine, contiene i valori di $\left(d T_{s} / d p_{s}\right)_{0}$ calcolati con la formula applossimata [3"].

L'accordo tra i risultati sperimentali e i valori teorici sembra abbastanza sorldisfarente.

\section{RLASSUNTO}

Tiene valutata, por via teorica, la variazione del punto di fusione con la pressione, per un solido ideale, a pressione ordinaria.

\section{ABSTRACT}

The rate of change of the melting point with pressure is derived on the basis of the classical theory of solids.

The values which have been obtained for Litium, Sodium, Potassium, and Iron, are compared with experimental data.

The resulting concordance is rather satisfactory.

\section{BIBIAOGRAFIA}

(1) Iswmasx, F. A., thber die Bereshnung molekulare Eigenfrequenzen. "Phys. Zs. ", 11. 609-612 (1910).

(2) Vande, P. E., Un'equazione di slato per i solidi. "Annali di Geofisica ", 6, 183 197 (1953).

$\left.{ }^{3}\right)$ - Luna stima del punlo di fasione del jerro sollo alle pressioni. "Annali di Geofisica ", $8,180-200(1955)$.

(1) Bridganan, P. W., The Physics of High Pressures. "Bell and sons", London, 1949.

$\left.{ }^{5}\right)$ - Llandbook of Chemistry and Physies. "('henical Rubber Publishingr Co.", Cleveland, 1957-58.

$\left.{ }^{6}\right)$ Sinon, F. E., The melling of iron al high pressures. "Nature ", 177, 746 (1953). 